\title{
Lacrimal Gland Carcinoma pT4a TNM Finding v8
}

National Cancer Institute

\section{Source}

National Cancer Institute. Lacrimal Gland Carcinoma pT 4a TNM Finding v8. NCI

Thesaurus. Code C140798.

Lacrimal gland carcinoma with involvement of adjacent structures, including sinuses,

temporal fossa, pterygoid fossa, superior orbital fissure, cavernous sinus, or brain and

measuring $2 \mathrm{~cm}$ or less in greatest dimension. (from AJCC 8th Ed.) 